\title{
Scolytinae y Platypodinae (Coleoptera: Curculionidae) de dos áreas de conservación en Tabasco, México
}

\author{
Manuel Pérez-De la Cruz ${ }^{1}$, Manuel A. Hernández-May ${ }^{1}$, Aracely De la Cruz-Pérez ${ }^{1 *}$ \\ \& Saúl Sánchez-Soto ${ }^{2}$ \\ 1. Universidad Juárez Autónoma de Tabasco, División Académica de Ciencias Biológicas, Código Postal 86150, \\ Carretera Villahermosa-Cárdenas km $0.5 \mathrm{~s} / \mathrm{n}$ entronque a Bosque de Saloya, Villahermosa, Tabasco, México; \\ arace_lycp@hotmail.com, perezmandoc@hotmail.com,sssoto@colpos.mx, albertomay040@gmail.com \\ 2. Colegio de Postgraduados, Campus Tabasco, Apartado postal 24, C. P. 86500, H. Cárdenas Tabasco, México; \\ sssoto@colpos.mx \\ * Correspondencia
}

Recibido 17-IX-2014. Corregido 24-IX-2015. Aceptado 26-X-2015.

\begin{abstract}
Scolytidae and Platypodidae (Coleoptera: Curculionidae) of two conservation areas in Tabasco, Mexico. The Scolytinae and Platypodinae are group of widely distributed tropical insects that are capable of using a wide range of hosts. The study of diversity associated with conservation sites allows estimating the richness and abundance of species, their population dynamics as well as understanding the population behavior over time. The species richness and abundance of Scolytinae and Platypodinae was studied in the Chontalpa Ecological Reserve (REC) and the José Narciso Rovirosa Botanical Garden (JBU) in Tabasco, Mexico. Insects were captured with ethyl alcohol traps. A total of 7057 specimens belonging to 46 species and 26 genera were collected and identified; 42 species from 23 genera belong to Scolytinae and four species from three genera to Platypodinae. The species Bothrosternus foveatus (Blackman, 1943); Dendroterus luteolus (Schedl, 1951); Dendrocranulus guatemalensis (Hopkins, 1915); Micracis swainei (Blackman, 1920); Pseudothysanoes tenellus (Wood, 1971) y Ambrosiodmus obliquus (Leconte, 1878) are new records for the state of Tabasco. The species Corthylus papulans Eichhoff, 1869 with 2509 specimens (35.55); Xyleborus volvulus (Fabricius, 1775) with 1435 (20.33); and Xyleborus affinis Eichhoff, 1868 with 1061 (15.03) were the most common species, representing $70.9 \%$ of total. The population dynamics of these insects showed its highest peak during February and March with 2728 specimens in JBU and December with 322 in REC of year 2010. Rev. Biol. Trop. 64 (1): 319-326. Epub 2016 March 01.
\end{abstract}

Key words: insects, bark beetles, population dynamics, Tabasco.

Las subfamilias Scolytinae y Platypodinae incluyen los comúnmente conocidos como escarabajos descortezadores y ambrosiales, son barrenadores de floema, xilema, médula de ramas y semillas de una amplia variedad de árboles, arbustos, hierbas y lianas. Algunas especies pueden atacar plantas vivas causando su muerte parcial o total, aunque la mayoría tienen preferencias por invadir plantas muertas o moribundas. Estos insectos se establecen sobre sus huéspedes con dos propósitos fundamentales; el de alimentarse y reproducirse (Burgos \& Equihua, 2007). La dinámica poblacional de estos insectos está influida por factores ambientales como temperatura, humedad, precipitación y bióticos como la competencia intra e inter específicas, parasitismo, depredación, respuesta genética y disponibilidad de alimento (Coulson, 1979); esta última característica se considera de las más importantes en la fluctuación poblacional de estos insectos (Morales, Zanuncio, Pratissoli, \& Fabres, 2000).

Ambas subfamilias son parecidas en cuanto a su morfología, ciclos biológicos, sistemas de reproducción y hábitos alimenticios, con base a esta última propiedad, se puede dividir 
a estos escarabajos como especies floeófagas, xilófagas, xilomicetófagas, herbífagas, mielofagas y espermatófagas (Equihua, Atkinson, \& Lott, 1984; Romero-Nápoles, Anaya, Equihua, \& Mejía, 1997; Baños-Juárez, Pérez-De la Cruz, Sánchez-Soto, \& Capello-García, 2012). Su papel principal en los ecosistemas naturales, se asocia con los procesos de descomposición de la madera (Equihua \& Burgos, 2002). Además son insectos que contribuyen con la estabilidad y en algunos casos con la inestabilidad de las comunidades vegetales, ya que algunas especies son plagas de árboles forestales (Atkinson \& Equihua, 1985).

De las 846 especies de Scolytinae registradas para México, menos del $5 \%$ son consideradas de importancia económica. Especies de los géneros Dendroctonus, Scolytus, Ips y Phloeosinus se admiten como plagas primarias, capaces de matar árboles sanos en bosques templados. Los géneros Gnathotrichus, Xyleborus y Xylosandrus se destacan por su daño a la madera, influyendo de manera significativa en su calidad. La broca del café Hypothenemus hampei (Ferrari, 1867) causa severos daños en diferentes variedades de café en varios estados del país (Equihua \& Brugos, 2002). Por tal motivo, nuestro objetivo es dar a conocer las especies de Scolytinae y Platypodinae asociados a la Reserva Ecológica de la Chontalpa y al Jardín Botánico José Narciso Rovirosa en Tabasco, México.

\section{MATERIALES Y MÉTODOS}

El estudio se realizó en la Reserva Ecológica de la Chontalpa (REC) y en el Jardín Botánico José Narciso Rovirosa (JBU) de febrero 2010 a enero 2011. La REC se localiza entre las coordenadas $\left(17^{\circ} 59^{\prime} 05^{\prime \prime} \mathrm{N}-93^{\circ} 34^{\prime} 13^{\prime \prime} \mathrm{W}\right)$ en el municipio de Cárdenas, Tabasco y cuenta con una extensión de 277 ha, presenta una vegetación de bosques tropical perennifolio siendo la especie dominante Bravaisia integerrima (Palma-López, García, Sol \& Juárez, 1999). El JBU, se encuentra dentro de las instalaciones de la División Académica de Ciencias Biológicas, ubicado dentro de las coordenadas
(17 59 '26” N - 92 58'16” W) en Villahermosa, Tabasco, con una superficie de 10 ha compuesta de vegetación secundaria (acahual) y porción de selva baja espinosa perennifolia de "tinto" (Haematoxylum campechianum) (Ascencio \& Maldonado, 2002). Ambas localidades registran precipitaciones anuales superiores a 2000 $\mathrm{mm}$, con clima Am (f), cálido húmedo con lluvias en verano y temperatura promedio anual de $27^{\circ} \mathrm{C}$ (Anónimo 2014).

Los insectos fueron capturados con siete trampas cebadas con alcohol etílico al $70 \%$ como atrayente, ubicadas en las áreas de mayor vegetación, a una altura de $1.50 \mathrm{~m}$, y a $50 \mathrm{~m}$ de distancia entre ellas distribuidas en línea recta. La recolección de los insectos se realizó quincenalmente, los especímenes se conservaron en alcohol al $70 \%$, para su posterior determinación y en cada recolección se procedió a reponer el alcohol (Bustamante \& Atkinson, 1984; Iturre \& Darchuck, 1996; Pérez-De la Cruz et al., 2009b).

Identificación del material biológico: La determinación taxonómica de los insectos la realizó el primer autor mediante el uso de claves taxonómicas (Wood, 1982; 1993, 1986; Pérez-De la Cruz, Equihua, Romero-Nápoles, Valdez, \& De la Cruz-Pérez, 2009a; Pérez-De la Cruz et al., 2011) se realizaron comparaciones con material depositado en la colección del Colegio de Postgraduados Campus Montecillo, México (CEAM), para corroborar la identificación de los individuos. Los especímenes se depositaron en la colección de insectos de la División Académica de Ciencias Biológicas de la Universidad Juárez Autónoma de Tabasco (CIUT, registro en trámite).

Análisis de datos: Para comparar la diversidad de Scolytinae y Platypodinae presentes en el JBU y REC se utilizó el índice de diversidad de Shannon-Wiener, que mide la estructura de la comunidad; el índice de riqueza de Margalef, el cual está basado en la riqueza específica; el índice de Pielou, el cual toma en cuenta la equidad de la comunidad; y el índice de similitud de Sorensen, basado en proporciones o 
diferencias, los cuales comúnmente son utilizados para el estudio de la diversidad de especies (Magurran, 1989; Moreno, 2001).

\section{RESULTADOS}

Se recolectaron 7057 especímenes pertenecientes a 46 especies incluidas en 26 géneros; del total de especies, 42 especies de 23 géneros pertenecieron a Scolytinae y cuatro especies de tres géneros a Platypodinae. Los géneros con mayor riqueza de especies fueron Hypothenemus con ocho especies y Xyleborus con seis. Las especies Corthylus papulans,
Xyleborus volvulus y $X$. affinis registraron la mayor abundancia en conjunto representan el $70.9 \%$ de la recolecta total (Cuadro 1).

El JBU presentó la mayor riqueza con 38 especies y una abundancia de 5818 organismos $(82.5 \%)$. Las especies C. papulans y $X$. vovulus registraron la mayor abundancia, en conjunto representaron el $62.2 \%$ de la recolecta total de esta zona. Dentro de la REC se obtuvo una menor riqueza con 27 especies y una abundancia de 1239 (17.5 \%). Las especies X. affinis y $X$. volvulus registraron la mayor abundancia de este sitio, y en conjunto representaron el 71.35 $\%$ de la colecta total.

CUADRO 1

Especímenes de Scolytinae y Platypodinae capturados en JBU y REC en Tabasco, México

TABLE 1

Specimens of Scolytinae and Platypodinae captured in REC and JBU in Tabasco, Mexico

\begin{tabular}{|c|c|c|c|c|}
\hline Scolytinae & JBU & REC & Total & $\%$ \\
\hline Ambrosiodmus obliquus (LeConte) 1878* & 2 & 0 & 2 & 0.02 \\
\hline Araptus sp. & 3 & 0 & 3 & 0.04 \\
\hline Bothrosternus foveatus (Blackman) 1943* & 0 & 1 & 1 & 0.01 \\
\hline Coccotrypes carpophagus (Hornung) 1842 & 0 & 1 & 1 & 0.01 \\
\hline Coccotrypes cyperi (Beeson) 1929 & 0 & 1 & 1 & 0.01 \\
\hline Corthylocurus debilis Wood 1974 & 307 & 1 & 308 & 4.36 \\
\hline Corthylus minutissimus Schedl 1940 & 7 & 80 & 87 & 1.23 \\
\hline Corthylus papulans Eichhoff 1869 & 2497 & 12 & 2509 & 35.55 \\
\hline Cryptocarenus diadematus Eggers 1937 & 37 & 0 & 37 & 0.52 \\
\hline Cryptocarenus heveae (Hagedorn) 1912 & 23 & 0 & 23 & 0.32 \\
\hline Cryptocarenus lepidus Wood 1971 & 49 & 2 & 51 & 0.72 \\
\hline Cryptocarenus seriatus Eggers 1933 & 10 & 0 & 10 & 0.14 \\
\hline Dendroterus luteolus (Schedl) 1951* & 1 & 0 & 1 & 0.01 \\
\hline Dendrocranulus guatemalensis (Hopkins) 1915* & 1 & 0 & 1 & 0.01 \\
\hline Dryocoetoides capucinus (Eichhoff) 1869 & 3 & 1 & 4 & 0.05 \\
\hline Micracis swainei Blackman 1920* & 4 & 0 & 4 & 0.05 \\
\hline Hypocryphalus mangiferae (Stebbing) 1914 & 3 & 0 & 3 & 0.04 \\
\hline Hypothenemus birmanus (Eichhoff) 1878 & 178 & 9 & 187 & 2.65 \\
\hline Hypothenemus brunneus (Hopkins) 1915 & 2 & 0 & 2 & 0.02 \\
\hline Hypothenemus crudiae (Panzer) 1791 & 80 & 3 & 83 & 1.17 \\
\hline Hypothenemus erectus LeConte 1885 & 1 & 0 & 1 & 0.01 \\
\hline Hypothenemus eruditus Westwood 1836 & 392 & 12 & 404 & 5.72 \\
\hline Hypothenemus interstitialis (Hopkins) 1915 & 4 & 0 & 4 & 0.05 \\
\hline Hypothenemus seriatus (Eichhoff) 1872 & 222 & 1 & 223 & 3.16 \\
\hline Hypothenemus areccae (Hornung) 1842 & 11 & 0 & 11 & 0.15 \\
\hline Premnobius cavipennis Eichhoff 1878 & 0 & 1 & 1 & 0.01 \\
\hline Pseudothysanoes tenellus Wood 1971* & 1 & 1 & 2 & 0.02 \\
\hline Sampsonius dampfi Schedl 1940 & 0 & 1 & 1 & 0.01 \\
\hline
\end{tabular}


CUADRO 1 (Continuación) / TABLE 1 (Continued)

\begin{tabular}{|c|c|c|c|c|}
\hline Scolytinae & JBU & REC & Total & $\%$ \\
\hline Stegomerus mexicanus Wood 1967 & 0 & 6 & 6 & 0.08 \\
\hline Stegomerus sp. & 6 & 0 & 6 & 0.08 \\
\hline Theoborus ricini (Eggers) 1932 & 126 & 0 & 126 & 1.78 \\
\hline Thysanoes mexicanus Wood 1956 & 8 & 0 & 8 & 0.11 \\
\hline Tricolus difodinus Bright 1972 & 10 & 1 & 11 & 0.15 \\
\hline Xyleborinus gracilis (Eichhoff) 1868 & 1 & 12 & 13 & 0.18 \\
\hline Xyleborus affinis Eichhoff 1868 & 375 & 686 & 1061 & 15.03 \\
\hline Xyleborus ferrugineus (F.) 1801 & 110 & 57 & 167 & 2.36 \\
\hline Xyleborus horridus Eichhoff 1869 & 2 & 0 & 2 & 0.028 \\
\hline Xyleborus posticus Eichhoff 1869 & 6 & 0 & 6 & 0.08 \\
\hline Xyleborus spinulosus Blandford 1898 & 0 & 91 & 91 & 1.28 \\
\hline Xyleborus volvulus (F.) 1775 & 1237 & 198 & 1435 & 20.33 \\
\hline Xylosandrus curtulus (Eichhoff) 1869 & 54 & 0 & 54 & 0.76 \\
\hline Xylosandrus morigerus (Blandford) 1894 & 1 & 7 & 8 & 0.11 \\
\hline \multicolumn{5}{|l|}{ Platypodinae } \\
\hline Euplatypus parallelus (F.) 1801 & 10 & 21 & 31 & 0.43 \\
\hline Euplatypus segnis (Chapuis) 1865 & 7 & 29 & 36 & 0.51 \\
\hline Teloplatypus excisus (Chapuis) 1865 & 27 & 3 & 30 & 0.42 \\
\hline Tesseroserus dawalquei Chapuis 1865 & 0 & 2 & 2 & 0.02 \\
\hline Total de especímenes & 5818 & 1239 & 7057 & 100 \\
\hline Total de especies & 38 & 27 & & \\
\hline
\end{tabular}

Lo señalado con* constituyen nuevos registros para Tabasco.

Las especies que se registraron con solo un espécimen fueron Dendroterus luteolus (Schedl, 1951); Dendrocranulus guatemalensis (Hopkins, 1915); Hypothenemus erectus LeConte, 1876, Xyleborinus gracilis (Eichhoff, 1868); Xylosandrus morigerus (Blandford, 1894); Bothrosternus foveatus (Blackman 1943); Coccotrypes carpophagus (Hornung, 1842); Coccotrypes cyperi (Beeson, 1929); Corthylocurus debilis Wood, 1974; Dryocoetoides capucinus (Eichhoff, 1869); Hypothenemus seriatus (Eichhoff, 1872); Premnobius cavipennis Eichhoff, 1878; Sampsonius dampfi Schedl, 1940 y Tricolus difodinus Bright, 1972.

De acuerdo con los índices de ShannonWiener y Margalef, la máxima diversidad de Scolytinae y Platypodinae capturados, se obtuvo en el JBU $\left(\mathrm{H}^{\prime}=1.94\right.$ y $\left.\mathrm{Dmg}=4.15\right)$, y la mínima en la REC $\left(\mathrm{H}^{\prime}=1.61\right.$ y $\left.\mathrm{Dmg}=3.51\right)$. Respecto al índice de equidad (J) el JBU obtuvo 0.54 y REC 0.50 ; la prueba estadística de $t$ a la diversidad $\left(\mathrm{H}^{\prime}\right)$ registró que ambos sitios presentaron diferencias estadísticamente significativas con $t_{0.05}$. Al determinar la similitud $\left(\mathrm{I}_{\mathrm{s}}\right)$ de especies de Scolytinae y Platypodinae presentes en el JBU y en REC, se determinó que ambos sitios comparten una diversidad de 19 especies con un valor de similitud de 0.603.

La fluctuación poblacional de los Scolytinae y Platypodinae presentó su máximo pico de recolecta durante febrero y marzo 2010 en el JBU. Dentro de la REC, el máximo pico de recolecta se registró en diciembre 2010, aunque con un número de organismo relativamente bajo comparado con el de JBU. Sin embargo, en la REC se observó que las poblaciones muestran estabilidad durante la mayor parte del periodo de estudio, es decir sus poblaciones se mantienen bajas (Fig. 1). Los máximos picos poblacionales registrados en febrero, marzo y diciembre coinciden con el periodo de temperaturas bajas y altos porcentajes de humedad en el JBU y la REC (Fig. 2A y Fig. 2B).

De acuerdo a la curva de acumulación de especies para JBU, no se logró colectar el máximo número de especies posibles con 


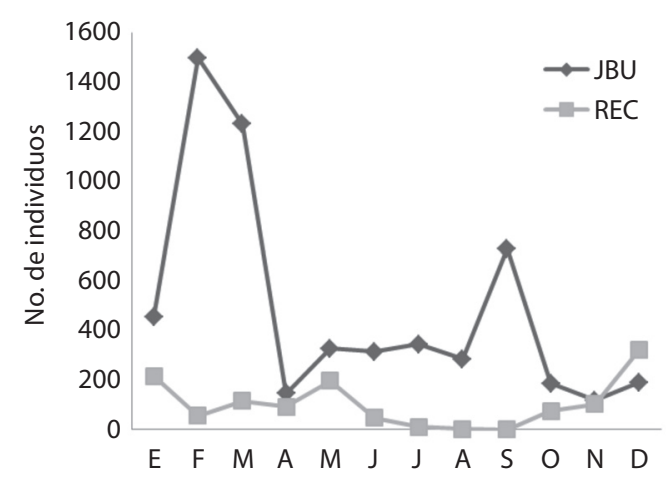

Fig. 1. Fluctuación poblacional de Scolytinae y Platypodinae capturados en JBU y REC de Tabasco, México.

Fig. 1. Population dynamics of Scolytinae and Platypodinae captured in REC and JBU of Tabasco, Mexico.
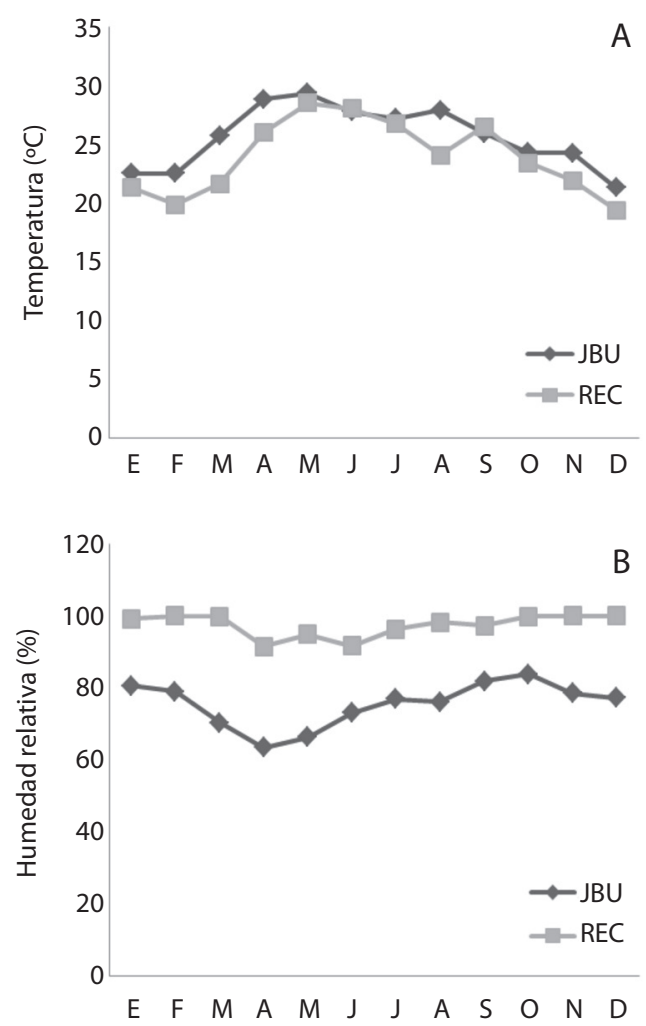

Fig. 2. A) Promedios mensuales de temperatura $\left({ }^{\circ} \mathrm{C}\right)$ registradas en JBU y REC en Tabasco, México. B) Porcentaje de humedad registrado en JBU y REC en Tabasco, México.

Fig. 2. A) Mean monthly temperature $\left({ }^{\circ} \mathrm{C}\right)$ recorded at JBU and REC in Tabasco, Mexico. B) Percentage of moisture registered JBU and REC in Tabasco, Mexico.

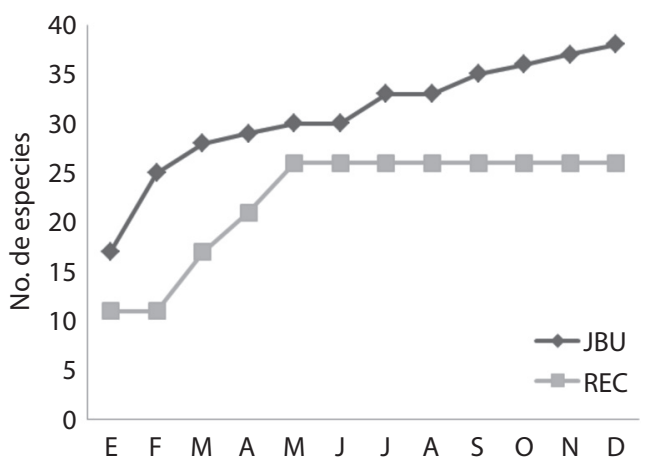

Fig. 3. Curva de acumulación de especies de Scolytinae y Platypodinae capturados en JBU y REC de Tabasco, México.

Fig. 3. Accumulation curve of Platypodinae and Scolytinae species captured in REC and JBU of Tabasco, Mexico.

el método de colecta utilizado, ya que no se alcanzó a estabilizar durante el tiempo que duró el estudio, lo que sugiere que todavía existen especies por colectar. A diferencia de la REC en donde la curva se estabilizó a partir del mes de mayo (Fig. 3).

\section{DISCUSIÓN}

Con base a los registros publicados por Schedl (1940), Equihua \& Burgos (2002), Pérez-De la Cruz et al., (2009b), Pérez-De la Cruz et al., (2009c), las especies B. foveatus, D. luteolus, D. guatemalensis, M. swainei, $P$. tenellus y $A$. obliquus constituyen nuevos registros para el estado de Tabasco, sumando un total de 68 especies de Scolytinae y seís de Platypodinae para el estado.

La riqueza de especies registrada por los géneros Hypothenemus y Xyleborus en el JBU y la REC coincide con lo reportado por PérezDe la Cruz et al., (2009b) en el agroecosistema cacao en Tabasco, México, en donde estos géneros han sido los más diversos. De igual manera, Atkinson \& Equihua (1986), reportan en un bosque tropical del sur de México al género Xyleborus con el mayor número de especies encontradas. Así mismo, Iturre \& Darchuck (1996) reportan a Hypothenemus y Xyleborus como los géneros más abundantes en especies 
asociadas a plantaciones de Eucalyptus en Santiago del Estero Argentina. Estos resultados se deben principalmente a que ambos géneros son de amplia distribución con alta tasa reproductiva, abundantes, y para los que la mayor diversidad de especies se ha registrado en regiones tropicales y subtropicales del mundo (Pérez-De la Cruz et al., 2009b).

$\mathrm{Al}$ igual que el género Xyleborus, la mayoría de las especies de Corthylus tienen una distribución tropical y muy pocas especies son consideradas plagas; sin embargo, algunas pueden causar la muerte o debilitamiento de ramas de perales, tejocote, manzano y membrillo (Bustamante \& Atkinson, 1984). La especie C. papulans ha sido reportada como la más abundante en este estudio con 2509 especímenes (35.55\%), así como, en el agroecosistema cacao en Tabasco (Pérez-De la Cruz et al., 2009b). Las altas poblaciones reportadas podrían estar dadas por la disponibilidad de los recursos vegetales y las condiciones ambientales que han permitido que se haya adaptado y sea una especie dominante (Estrada-Pérez, Pérez-De La Cruz, \& Hernández-May, 2012). Además, la especies $X$. volvulus con 1435 especímenes (20.33\%) y X. affinis con 1061 $(15.03 \%)$ se reportaron como las especies más abundantes; en México X. volvulus es considerada una especie de importancia económica en las áreas tropicales, debido a su amplia distribución y capacidad de colonizar plantas sanas, dado que se encuentra asociada a la muerte del árbol de sombra (Erythrina americana Miller) del cacao, además de infestar madera de Cedrela odorata (L.) (Cibrián-Tovar, Méndez, Campos, Yates III, \& Flores, 1995). Es una especie polífaga asociada a más de 122 especies de plantas (Wood, 1982). La especie X. affinis también es conocida por su amplia distribución pantropical e inclusive por ser una especie cosmopolita, reportada en alrededor de 300 huéspedes. La alta capacidad para colonizar una lista considerable de plantas hace que estas especies puedan tener grandes anternativas para mantener poblaciones elevadas.

La máxima diversidad registrada en el JBU se debe a las diferencias existentes en el número de especies y su abundancia, en comparación a las registradas en la REC; sin embargo, la mayoría de las especies de Scolytinae y Platypodinae tienden a ser igualmente abundantes, a pesar de que hay especies como C. papulan, $X$. affinis y $X$. vovulus que se registraron como especies dominantes, debido al número de especímenes recolectados.

Las diferencias encontradas en la riqueza y abundancia de estos insectos en las dos localidades estudiadas, podrían estar dadas por las características particulares de cada sitio (como la diversificación vegetal, disponibilidad de alimento, edad de las plantas, competidores, depredadores, aunados con la temperatura y humedad), debido a que tanto factores bióticos como abióticos pueden influir en las poblaciones de estos insectos (Rudinsky, 1962; Wood, 1982; Pérez-De la Cruz et al., 2009b). Aunado a ello en el JBU, los árboles están más dispersos, mientras que en la REC los árboles se presentan en una alta densidad y proporcionan una mayor cantidad de sombra, además de que funcionan como barreras que impiden la dispersión del material atrayente. Otro factor que puede influir en la abundancia de estos insectos es el daño causado por la radiación solar en las ramas de los árboles, la cual es más notable en áreas donde la vegetación se encuentra más dispersa.

La fluctuación y los máximos picos poblacionales de Scolytinae y Platypodinae reportados en este estudio son similares a lo encontrado en el agroecosistema cacao en Tabasco, determinando que a inicio (febrero-marzo) y al final del año (septiembre-diciembre) se registran las mejores condiciones donde estos insectos alcanzan sus máximas poblaciones, ya que, estos escarabajos están asociados a hongos íntimamente ligados a la disponibilidad de alimento con humedad y temperatura óptima para su establecimiento, lo cual se presenta durante esta temporada del año (Pérez-De la Cruz et al., 2009b; Rangel, Pérez, Sánchez, \& Capello, 2012; Estrada-Pérez et al., 2012). Además, se observó que los Scolytinae y Platypodinae se mantienen activos durante todo el periodo de 
estudio, aunque en la mayor parte del año sus poblaciones se encuentran bajas.

En el JBU, la curva de acumulación de especies no llegó a estabilizarse, lo que indica que hay especies que no fueron capturadas, por lo que se recomienda utilizar otros métodos de recolecta complementarios que coadyuven a capturar el mayor número de especies posibles. Aunque, las trampas de alcohol son un método de recolecta eficiente para Scolytinae y Platypodinae, existen otros métodos, como son las trampas de luz, plantas trampas y la recolecta directa, los cuales han demostrado ser complementarios para obtener la mayor diversidad de estos insectos en una región determinada (Pérez-De la Cruz et al., 2009b).

\section{AGRADECIMIENTOS}

A los revisores anónimos y a Ena E. Mata Zayas por las aportaciones hechas al manuscrito.

\section{RESUMEN}

Los Scolytinae y Platypodinae son insectos tropicales de amplia distribución, capaces de utilizar una amplia gama de huéspedes. El estudio de la diversidad asociados a sitios de conservación nos permite conocer la riqueza y abundancia de las especies, además su fluctuación poblacional ayuda a entender el comportamiento de las poblaciones a través del tiempo. Por tal motivo, la riqueza y abundancia de las especies de Scolytinae y Platypodinae fue estudiada en la Reserva Ecológica de la Chontalpa (REC) y el Jardín Botánico José Narciso Rovirosa (JBU), en Tabasco, México. Los insectos fueron capturados con trampas de alcohol etílico. Se recolectaron 7057 especímenes pertenecientes a 46 especies incluidas en 26 géneros, de las cuales 42 especies de 23 géneros pertenecen a Scolytinae y cuatro especies de tres géneros a Platypodinae. Las especies $B$. foveatus, D. luteolus, D. guatemalensis, Micracis swainei (Blackman, 1920), Pseudothysanoes tenellus (Wood, 1971) y Ambrosiodmus obliquus (Leconte, 1878) constituyen nuevos registros para el estado de Tabasco. Las especies Corthylus papulans Eichhoff, 1869 con 2509 especímenes (35.55\%), Xyleborus volvulus (Fabricius, 1775) con 1435 (20.33\%) y Xyleborus affinis Eichhoff, 1868 con 1061 $(15.03 \%)$ registraron la mayor abundancia, que en conjunto representan el $70.9 \%$ de la recolecta total. La fluctuación poblacional de estos insectos presentó su máximo pico durante febrero y marzo con 2728 especímenes en el JBU $y$ en la REC en diciembre con 322 en el 2010.
Palabras clave: insectos, barrenadores, fluctuación poblacional, Tabasco.

\section{REFERENCIAS}

Anónimo. (2014). Instituto Nacional de Estadística Geografía e Informática (INEGI). Retrieved from http:// cuentame.inegi.org.mx.

Ascencio, R. J. M. \& Maldonado M. F. (2002). El Jardín Botánico Universitario "José Narciso Rovirosa" un esfuerzo para la conservación de la flora tabasqueña. Revista de Divulgación Kuxulkab, 7, 34-47.

Atkinson, T. H., \& Equihua, M. A. (1985). Lista comentada de los coleópteros Scolytidae y Platypodidae del valle de México. Folia Entomológica Mexicana, 65, 63-108.

Atkinson, T. H., \& Equihua, M. A. (1986). Biology of bark and ambrosia beetles (Coleoptera: Scolytidae and Platypodidae) of a tropical rain forest in southeastern Mexico with an annotated checklist of species. Annals of the Entomological Society of America, 79, 414-423.

Baños-Juárez, A., Pérez-De la Cruz, M., Sánchez-Soto, S., \& Capello-García, S. (2012). Fluctuación poblacional de Xyleborus volvulus (F.) (Coleoptera: Curculionidae) en localidades de Tabasco, México. Acta Zoológica Mexicana (n.s.), 28, 540-549.

Burgos, S. A. \& Equihua, M. A. (2007). Platypodidae y Scolytidae (Coleoptera) de Jalisco, México. Dugesiana, 14, 59-82.

Bustamante, O. F. \& Atkinson, H. T. (1984). Biología del barrenador de las ramas del peral Corthylus fuscus Blandford (Coleoptera: Scolytidae), en el norte del Estado de Morelos. Folia Entomológica Mexicana, 60, 83-101.

Cibrián-Tovar, D., Méndez, M. J. T., Campos, B. R., Yates, III H. O., \& Flores, L. J. (1995). Insectos Forestales de México/Forest Insects of Mexico. México: Universidad Autónoma Chapingo.

Coulson, R. N. (1979). Population dynamics of bark beetles. Annual Review of Entomology, 24(1), 417-47.

Equihua, M. A., Atkinson,H. T., \& Lott, E. (1984). Scolytidae y Platypodidae (Coleoptera) de la estación de Biología Chamela, Jalisco. Agrociencia, 57, 179-193.

Equihua, M. A. \& Burgos, S. A. (2002). Scolytidae. In B. J. Llorente \& J. J. Morrone (Eds.), Biodiversidad, Taxonomía y Biogeografía de artrópodos de México: Hacia una sintesis de su conocimiento (Vol. III, pp. 539-557). México: CONABIO-IBUNAM.

Estrada-Pérez, N., Pérez-De La Cruz, M., \& HernándezMay, M. A. (2012). Fluctuación poblacional de Corthylus spp. (Coleoptera: Curculionidae) en Tabasco, 
México. Boletin del Museo de Entomología de la Universidad del Valle, 13, 16-24.

Iturre, M., \& Darchuck, E.(1996). Registros de escolítidos relacionados al género Eucalyptus en Santiago del Estero. Quebracho, 4, 11-9.

Magurran, E. A. (1989). Diversidad ecológica y su medición. Barcelona: Vedra.

Morales, N. E., Zanuncio, J. C., Pratissoli, D., \& Fabres, A. S. (2000). Fluctuación poblacional de Scolytidae (Coleoptera) en zonas reforestadas con Eucalyptus grandis (Myrtaceae) en Minas Gerais, Brasil. Revista Biología Tropical, 48(1), 101-107.

Moreno, C. E. (2001). Métodos para medir la biodiversidad (vol. 1). Zaragoza: SEA.

Palma-López, D. J., García, L. E., Sol, S. A., \& Juárez, L. F. (1999). Plan de manejo de la Reserva Ecológica de la Chontalpa. México: Colegio de Postgraduados, Campus Tabasco y SEDESPA.

Pérez-De la Cruz, M., Equihua, M. A., Romero-Nápoles, J., Valdez, C. J. M., \& De la Cruz-Pérez, A. (2009a). Claves para la identificación de escolitinos (Coleóptera: Curculionidae: Scolytinae) asociados al agroecosistema cacao en el sur de México. Boletín del Museo de Entomología de la Universidad del Valle, 10(1), 14-29.

Pérez-De la Cruz, M., Equihua, M. A., Romero-Nápoles, J., Sánchez-Soto, S., \& García-López, E. (2009b). Diversidad, fluctuación poblacional y plantas huésped de escolitinos (Coleoptera: Curculionidae) asociados con el agroecosistema cacao en Tabasco, México. Revista Mexicana de Biodiversidad, 80, 779-791.

Pérez-De la Cruz, M., Equihua, M. A., Romero-Nápoles, J., Sánchez-Soto S., García-López, E., \& Bravo, M. H. (2009c). Escolítidos (Coleoptera: Scolytidae)
Asociados al Agroecosistema Cacao en Tabasco, México. Neotropical Entomology, 38(5), 602-609.

Pérez-De la Cruz, M., Valdez, C. J. M., Romero-Nápoles, J., Equihua, M. A., Sánchez-Soto, S., \& De la CruzPérez, A. (2011). Fluctuación poblacional, plantas huéspedes, distribución y clave para la identificación de Platypodinae (Coleoptera: Curculionidae) asociados al agroecosistema cacao en Tabasco, México. Acta Zoológica Mexicana (n.s.), 27(1), 129-143.

Rangel, R., Pérez, M., Sánchez, S., \& Capello, S. (2012). Fluctuación poblacional de Xyleborus ferrugineus y $X$. affinis (Coleoptera: Curculionidae) en ecosistemas de Tabasco, México. Revista de Biología Tropical, 60(4), 1577-1588.

Romero-Nápoles, J., Anaya, R. S., Equihua, M. A., \& Mejía, G. H. (1997). Lista de Scolytidae y Platypodidae de México (Insecta: Coleoptera). Acta Zoológica Mexicana (n.s), 70, 35-53.

Rudinsky, L. A. (1962). Ecology of Scolytidae. Annual Review of Entomology, 7, 327-348.

Schedl, K. E. (1940). Scolytidae, Coptonotidae y Platypodidae mexicanos. Anales de la Escuela Nacional de Ciencias Biológicas Instituto Politécnico Nacional, 1, 317-378.

Wood, S. L. (1982). The bark and ambrosia beetles of North and Central America (Coleoptera: Scolytidae), a taxonomic monograph. Great Basin Naturalist Memoirs, 6, 1-1327.

Wood, S. L. (1986). A reclassification of the genera of Scolytidae (Coleoptera). Great Basin Naturalist Memoirs, 10, 1-126.

Wood, S. L. (1993). Revision of the genera of Platypodidae (Coleoptera). Great Basin Naturalist Memoirs, 53, 259-281. 